\title{
Hadronic Calibration of the ATLAS Calorimeter
}

Rolf Seuster

University of Victoria

CALOR'06

$$
5^{\text {th }}-9^{\text {th }} \text { June } 2006
$$

for the ATLAS Hadronic Calorimeter Calibration Group

\section{Introduction}

Jet Energy Scale / Local Hadronic Calibration

Dead Material Correction

Conclusion 


\section{Introduction}

- Jets important ingredient for many physics analysis:

QCD, top, searches, Higgs, ...

- jets made out of hadrons in ATLAS response different than for electrons!

- non-compensating calorimeters in ATLAS require software calibration of calorimeter 


\section{Introduction, cont'd}

- precision physics at LHC :

top mass to $1 \mathrm{GeV} \quad(\sim 0.5 \%)$

PDG(2005)

$\mathrm{W}$ mass to $20 \mathrm{MeV}(\sim 0.025 \%) \quad 80.425 \pm 0.038 \mathrm{GeV}$

- precise knowledge of response of calorimeters necessary

$$
\mathrm{Z}_{0}->\mathrm{e}^{+} \mathrm{e}^{-} @ \sim 1 \mathrm{~Hz}
$$

- precise knowledge of response of calorimeters to hadrons necessary

no obvious process for calibration,

top, dijets for cross checking the calibration 


\section{Mass of the Top Quark}

- best current value (preliminary) :

$$
172.5 \pm 1.3 \text { (stat) } \pm 1.9 \text { (syst) } \mathrm{GeV} / \mathrm{c}^{2}
$$

- $1.3 \%$ precision !!

- Tevatron average (see hep-ex/0603039)

- already now, systematics dominated!

- dominant error:

Jet Energy Scale
Mass of the Top Quark ( ${ }^{*}$ Preliminary) Measurement

\begin{tabular}{|c|c|}
\hline DF-I & \\
\hline$\varnothing-1$ & di \\
\hline$D F-I I$ & \\
\hline$\varnothing-11$ & \\
\hline DF-I & \\
\hline ) & \\
\hline CDF-II & $\mathrm{I+}$ \\
\hline$D \varnothing-I I$ & \\
\hline$F_{1}$ & \\
\hline
\end{tabular}

Tevatron Run-I/I*

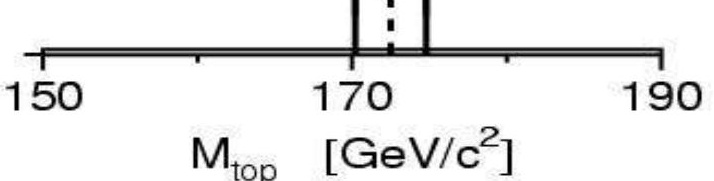

$M_{\text {top }}\left[\mathrm{GeV} / \mathrm{c}^{2}\right]$

$167.4 \pm 11.4$

$168.4 \pm 12.8$

$164.5 \pm 5.5$

$176.6 \pm 11.8$

$176.1 \pm 7.3$

$180.1 \pm 5.3$

$173.4 \pm 2.8$

$170.6 \pm 4.6$

$186.0 \pm 11.5$

$\chi^{2} /$ dof $=8.1 / 8$

$172.5 \pm 2.3$ 


\section{Jet Energy Scale}

'convential' scheme

Detector response

EM objects

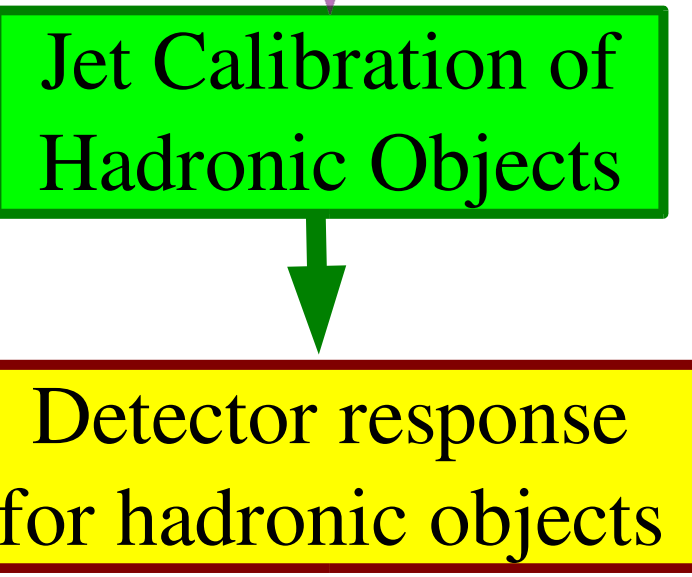

ATLAS scheme

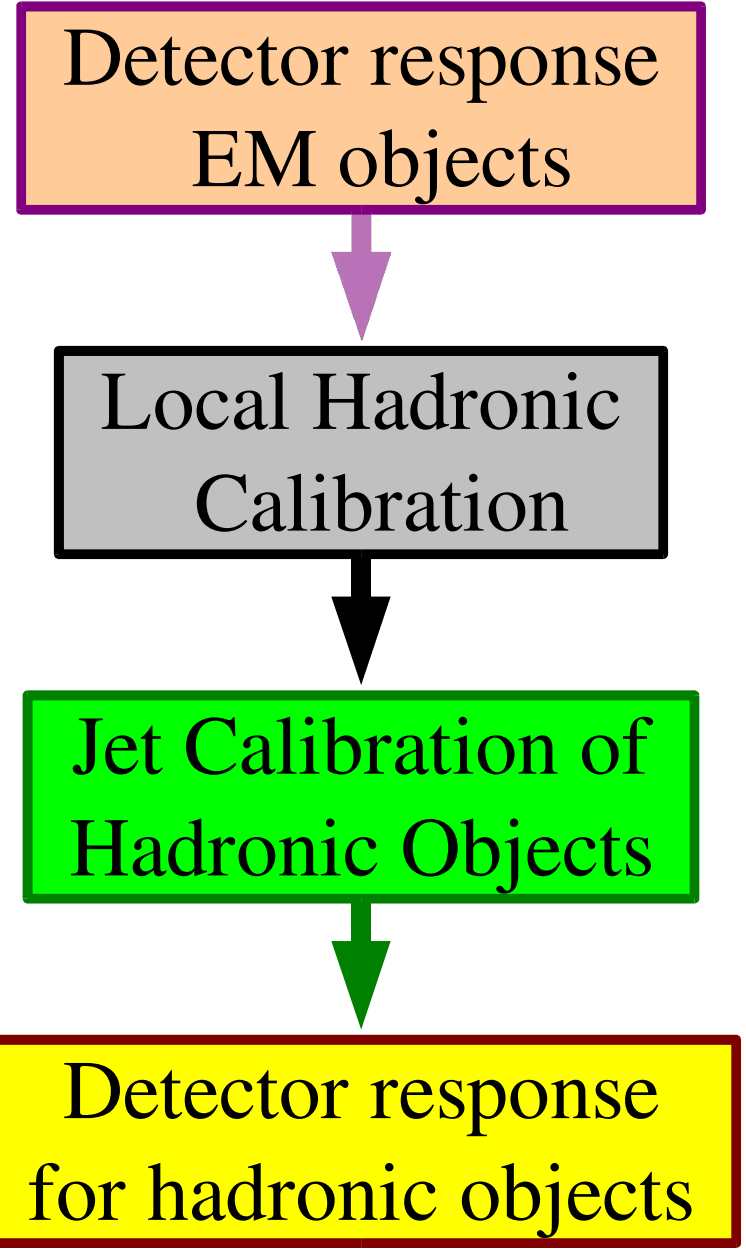




\section{Jet Energy Scale}

'convential' scheme

Detector response

EM objects

Jet Calibration of

Hadronic Objects

$\checkmark$

Detector response for hadronic objects
ATLAS scheme

Detector response

EM objects

calorimeter dependent

physics dependent

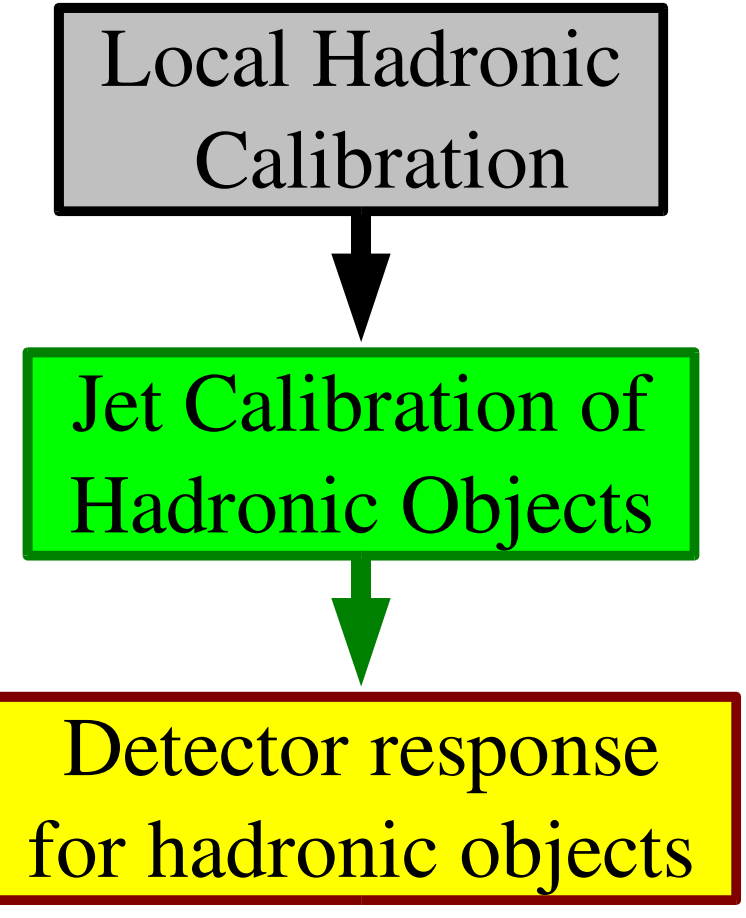




\section{Local Hadronic Calibration}

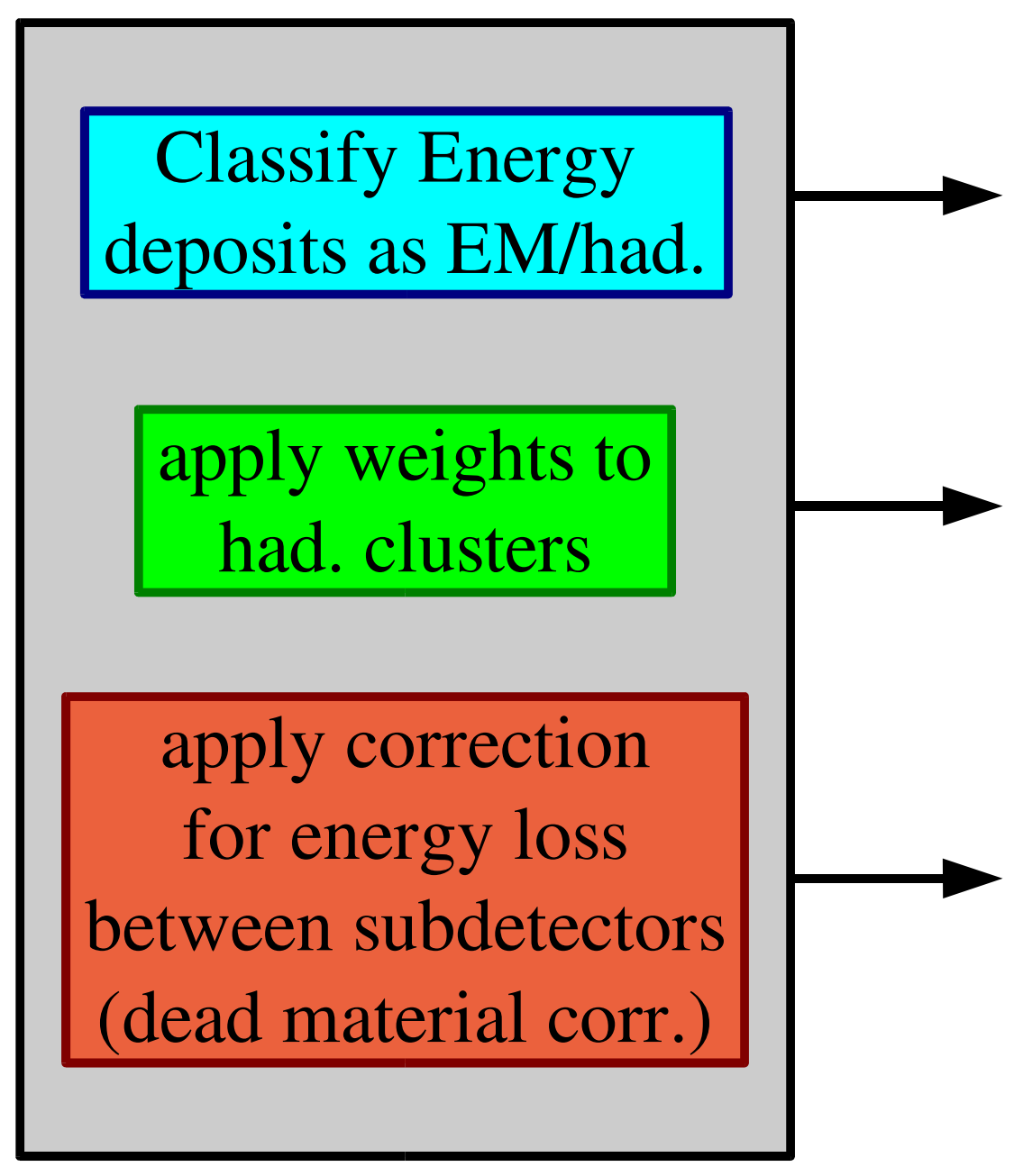

Which cluster need correction for hadronic activity?

determine correct energy response for hadrons relative to electrons

correct for leakage and energy loss inside and between subdetectors 


\section{Jet Energy Scale in ATLAS}

- ingredients to calibrate the jet energy scale:

- build clusters of neighbouring calorimeter cells

- "cluster classification" and apply correction accordingly

- calibrate energy in these clusters ('energy blobs') as good as possible "w eights"

- then, go to physics objects : 'jets' possibly apply second correction 


\section{Building Cluster}

- several different clusters in ATLAS:

Region-of-Interest (trigger),

Sliding Window (e/ $/$ ), ...

- mostly used :

'C aloTopoCluster" topological clustering several options:

-seeds, which neighbours to include $(2 \mathrm{~d}, 3 \mathrm{~d})$

-cluster also over subdetector boundaries, ... 


\section{"CaloTopoCluster"}

- 3 main variables determine the algorithm

- $\underline{\text { seed }}:$ default $4 \sigma$ of total noise

(elec. $\oplus$ pileup)

- $\underline{\text { neighbour }}: \square$ default $2 \sigma$

- $\underline{\text { cell }}: \square$ default $0 \sigma$

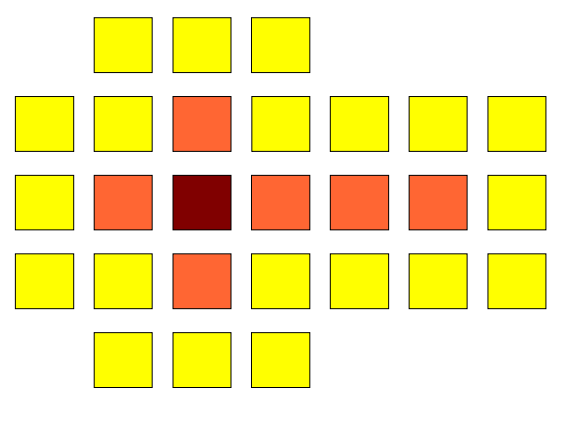

- can span over subdetectors 


\section{Splitting the Clusters}

- objects closeby can have overlaping clusters

$\rightarrow$ need to split clusters

- splitter looks for local maxima inside a cluster one cell can share energy between two clusters

- objective:

- electrons contained in one cluster

- pions can deposit energy in several clusters 


\section{Energy Scale: EM}

- EM scale is basic scale to start from, possibly corrected for well known HV problems

- Liquid Argon Calorimeters - day 0: comparison test beam - MC

- Tile Calorimeter - day 0: ${ }^{137} \mathrm{Cs}$ intercalibration and test beam

- after few days of running, $\mathrm{Z} \rightarrow \mathrm{e}^{+} \mathrm{e}^{-}$allows cross check of calibration, refinement possible goal permille level 


\section{Cluster Classification}

- determine correct weights for different types of clusters : electromagnetic, hadronic, muonic, ...

- currently based on two observables:

-longitudinal maximum of energy deposit: $\lambda$

-average energy density:

- cut on e.m. fraction of a cluster, determined from single pion $\mathrm{MC}$ : 


\section{Hadronic Energy Scale: Classification of EM Clusters}

\section{$\ldots>$ Cluster Moments $>\left\langle\rho_{\text {cell }}\right\rangle$}

- $\left\langle\rho_{\text {cell }}\right\rangle$ : energy weighted average (first moment) of cell energy densities inside the cluster for $200 \mathrm{k}$ single pions from $3 \mathrm{GeV}$ to $1 \mathrm{TeV}$

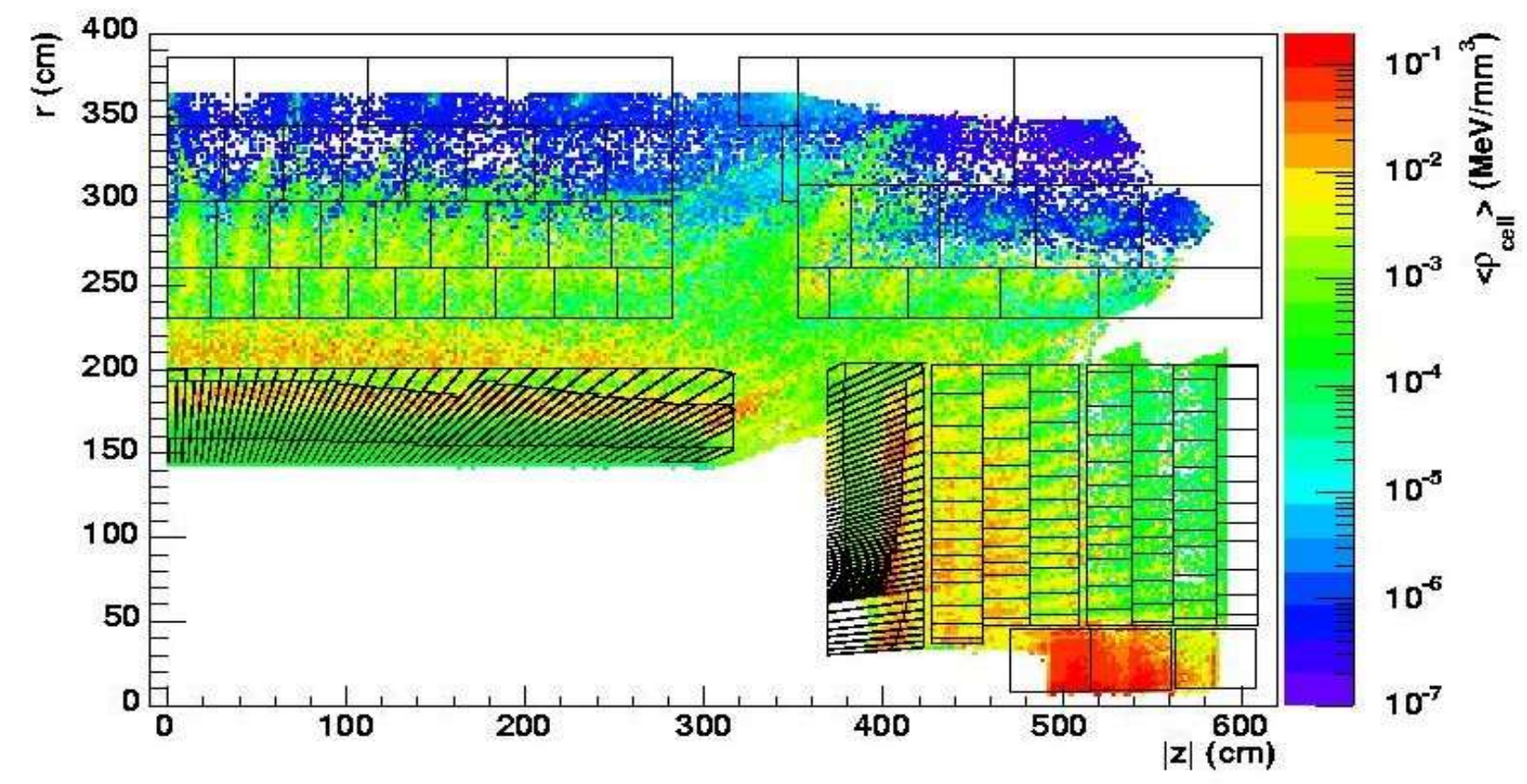




\section{Hadronic Energy Scale: Classification of Clusters}

- classify as em, hadron or muon based on cluster shapes (e.g. average density, shower max., etc.)

- compare test beam data and MC:
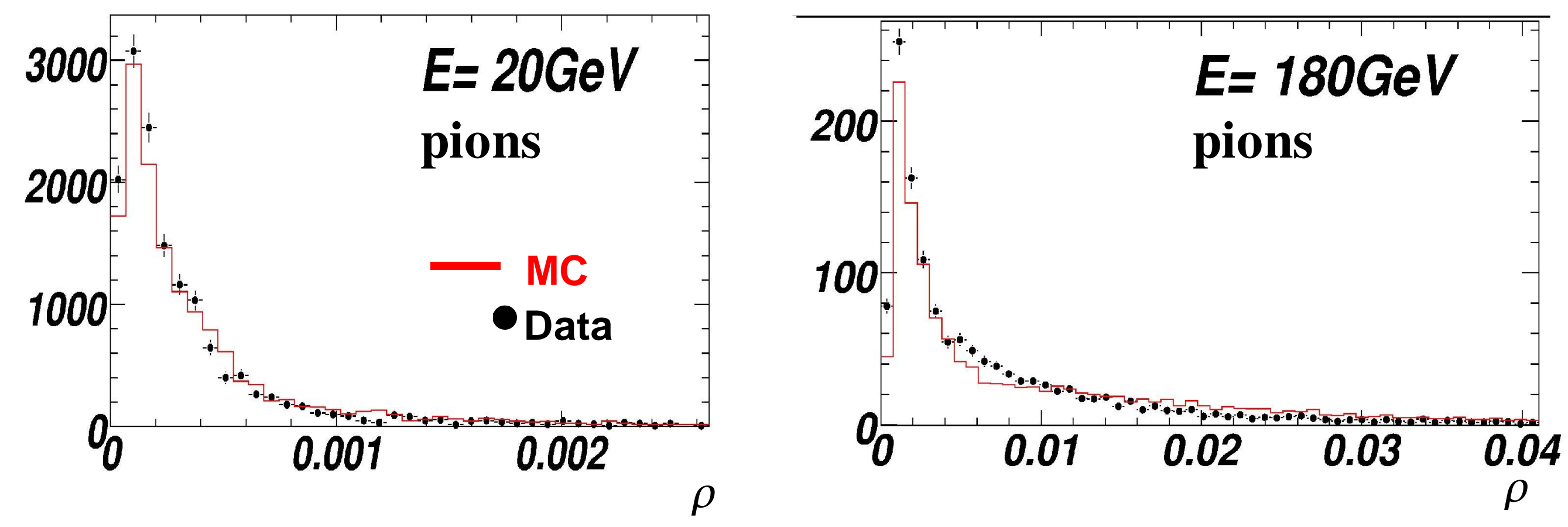


\section{Hadronic Energy Scale: Weights}

- weights based on MC :so called 'Calibration hits' each GEANT4 hit (energy deposit) classified as:

-EM energy $\quad(\sim 50 \%)$

-non-EM energy $(\sim 25 \%)$

-invisible energy $(\sim 25 \%)$

-escaped energy $\quad(\sim 2 \%)$

- fractions vary with particle's energy

- large fluctuations

\section{Electromagnetic Energy}




\section{Hadronic Energy Scale: Weights ${ }^{\text {contd }}$}

- weights:

$$
\begin{aligned}
E_{C E L L}^{\text {reco }} & =w(\vec{x}) E_{C E L L}^{\text {raw }} \\
w(\vec{x}) & =\frac{\left\langle E_{E M}+E_{\text {non-EM }}+E_{\text {invisible }}+E_{\text {escaped }}\right\rangle}{\left\langle E_{E M}+E_{\text {non-EM }}\right\rangle}
\end{aligned}
$$

- depending on several parameters $\vec{x} \quad \rightarrow$ next slide

- can include dead material inside cluster

- introduces MC dependence into calibration some sensitivity on modelling hadronic showers 


\section{Hadronic Energy Scale: Weighting Schemes}

- different weighting schemes under investigation:

- current standard (by S. Menke):

$$
w(\vec{x}) \equiv w\left(E_{\text {Cluster }}, \rho_{\text {Cell }}\right)
$$

- alternative approach (C. Issever \& Stockholm group):

$$
w(\vec{x}) \equiv w\left(E_{\text {global }}, \rho_{\text {Cell }}\right)
$$

\section{$\mathrm{E}_{\text {global }}$ : energy in cone (11 degrees)}




\section{Dead Material Correction}

- last important ingredient:

energy deposits in dead material

need MC with calibration hits for correcting this

- super-3D Topocluster span over different calorimeters:

energy deposits between (before) subdetectors

- correction relies on good description of detector

- EMB0
EMB1
EMB3_TILEO
- LEAK
KNOWN

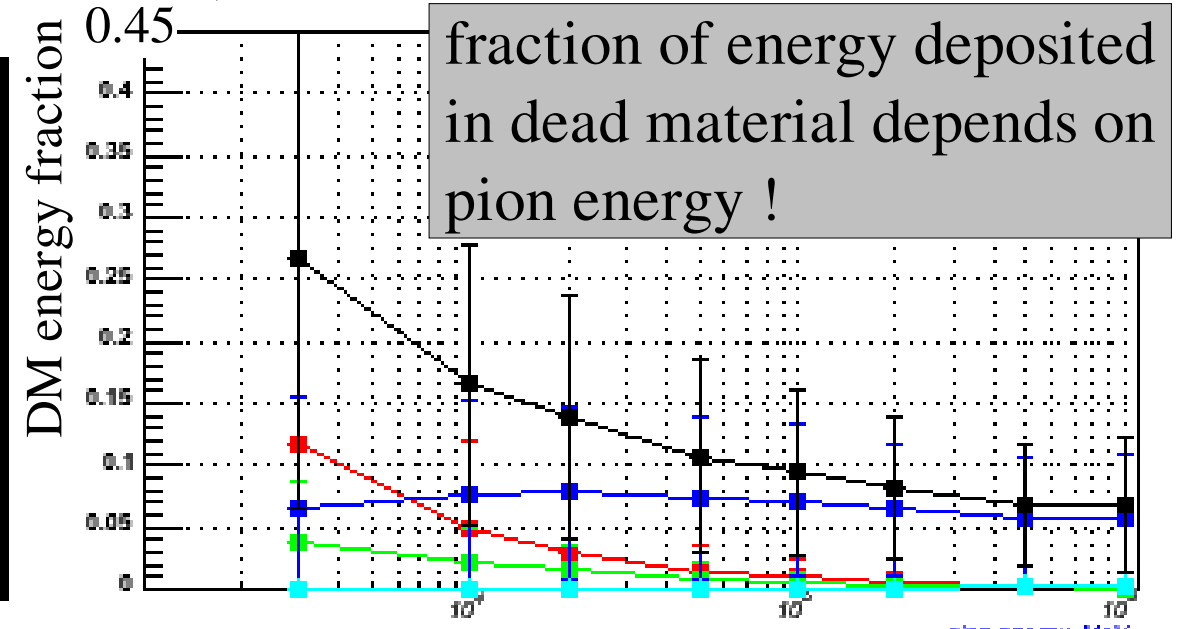




\section{Dead Material Corrections}

DM energy in different zones for 20 and $100 \mathrm{GeV}$ pi-.

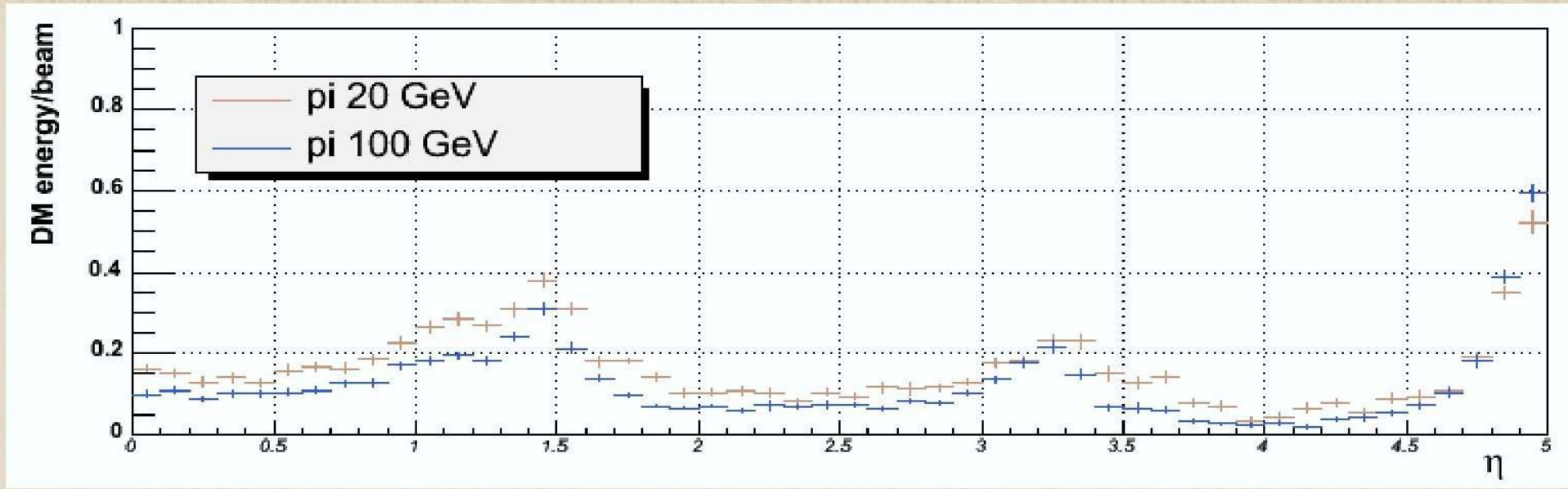

Ratio of total DM energy to the beam energy as a function of particle eta.

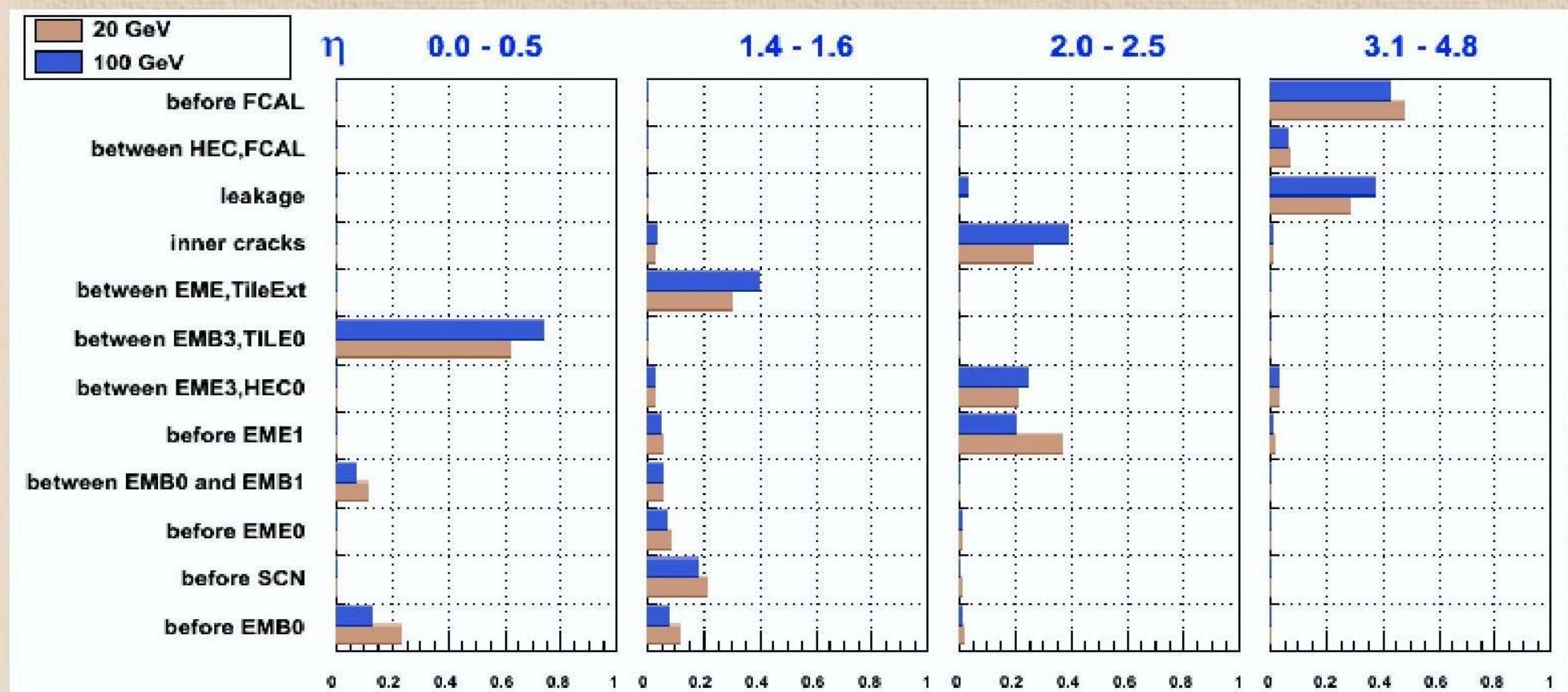

Ratio of DM energy released in particular zone to full DM energy accumulated in container. Four different eta areas are shown. 


\section{Adding all together ...}

applying to QCD di-jets, only 2 highest jets shown here

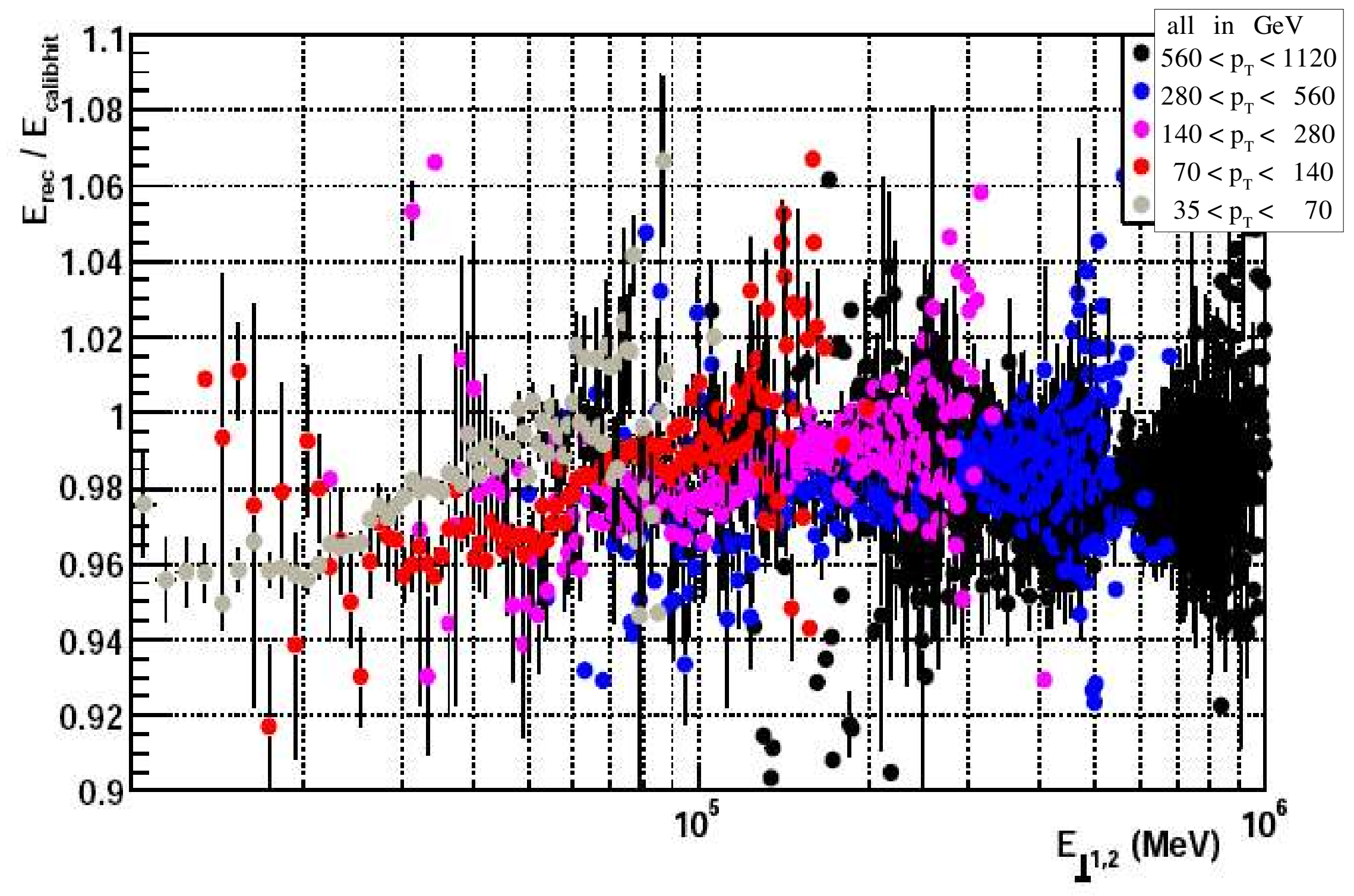




\section{Improvement in Resolution}

\section{Performance on Dijets $>$ Resolution}

- leading 2 jets in J4 sample with truth matching

- select $1.9<|\eta|<2.3$

$\checkmark$ plot $E / E_{\text {truth }}$ for raw (red), weighted (blue) and true calibration hits (green)

- scale is correct to $98 \%$ after weighting

- resolution improves from $5.9 \%$ to $4.9 \%$ (theoretically achievable is $4.0 \%$ )

\section{VERY PRELIMINARY}

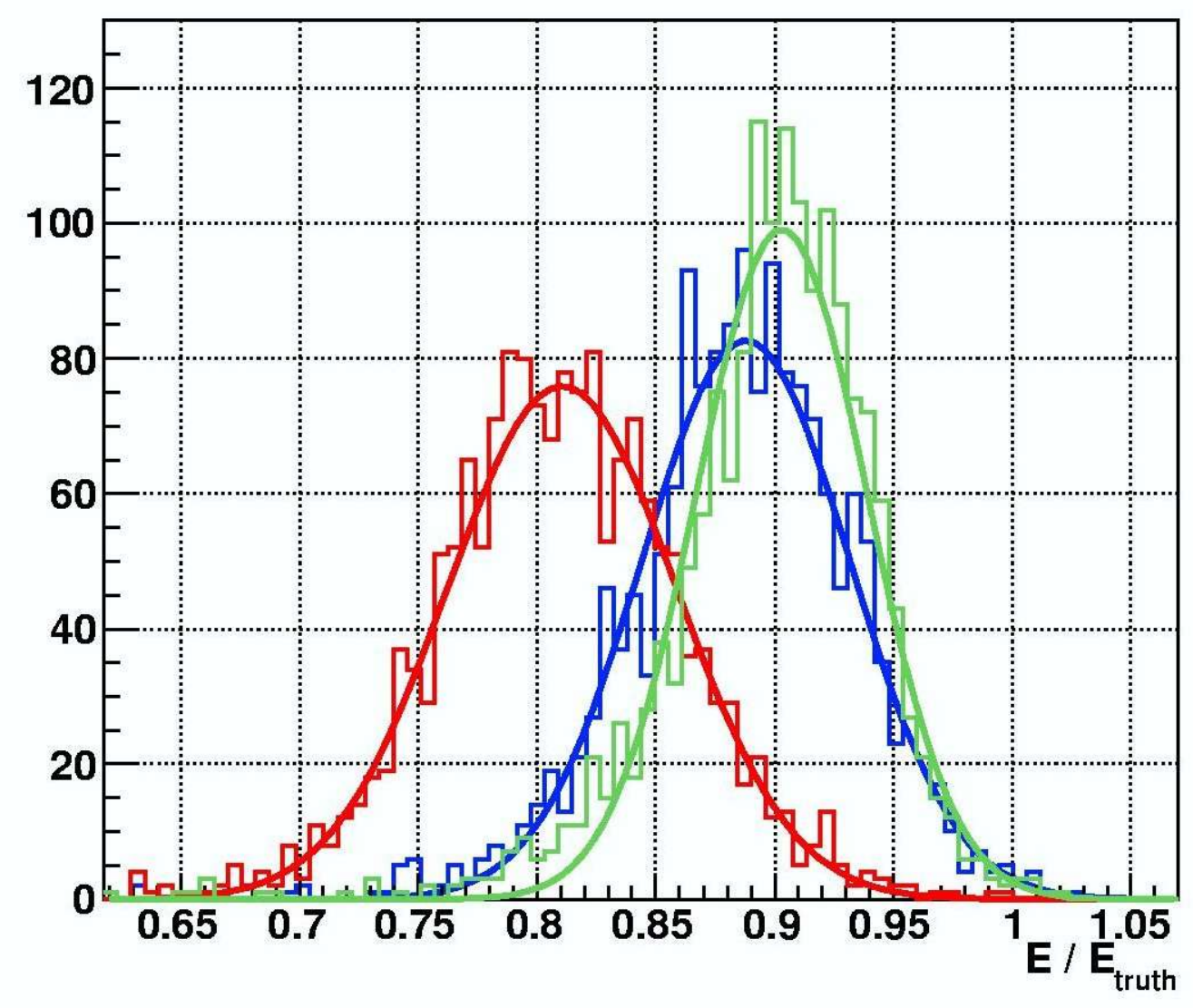




\section{Conclusions}

- idea and current status of local hadronic calibration of ATLAS Calorimeter presented

- first very preliminary results show good behaviour :

\section{improvement in resolution}

